\title{
Dimethylformamide Dimethyl Acetal (DMFDMA) in Heterocyclic Synthesis: Synthesis of Polysubstituted Pyridines, Pyrimidines, Pyridazine and Their Fused Derivatives
}

\author{
Fathi A. Abu-Shanab ${ }^{1 *}$, Sayed A. S. Mousa ${ }^{1}$, E. A. Eshak ${ }^{1}$, Ahmed Z. Sayed ${ }^{2}$, Ahmed Al-Harrasi ${ }^{3}$ \\ ${ }^{1}$ Department of Chemistry, Faculty of Science, Al-Azhar University, Assiut, Egypt \\ ${ }^{2}$ Chemistry Department, Umm Al-Qura University, Makkah Al-Mokaramah, Saudia Arabia \\ ${ }^{3}$ Department of Biological Sciences and Chemistry, College of Arts and Sciences, University of Nizwa, \\ Nizwa, Sultanate of Oman \\ E-mail: "fathiabushanab@yahoo.com
}

Received September 29, 2011; revised November 5, 2011; accepted November 13, 2011

\begin{abstract}
Reaction of $N, N^{\prime}$-dimethylformamide dimethyl acetal (DMFDMA) with malononitrile dimer 8 (1:1) mole afforded $\mathbf{9}$ while, this reaction when carried out in (2:1) mole to give amidine $\mathbf{1 1}$ which can be used for the preparation of pyrimidine 13, amidine 14 and pyridine 19 when reacted with 4-nitroaniline, 4-methylaniline and alkoxide respectively. Malononitrile dimer reacted with diazonium chloride to give pyridazine 21, which can be reacted with DMFDMA, $\mathrm{AcOH} / \mathrm{HCl}$ and cyanoacetamide to give pyridazine 22, 23 and pyrido[4,3-c] pyridazine 24 respectively. The latter reacted with DMFDMA to afford tricyclic compound 25.
\end{abstract}

Keywords: DMFDMA, Malononitrile Dimer, Pyridazine-3,5-carbonitrile, Pyridine-4-alkoxide

\section{Introduction}

Formamide acetals are useful reagents in organic synthesis; $[1,2]$ their main application has been used for functional group transformations [3], but they may also be regarded as one-carbon synthons in the construction of carbon skeletons. One type of reaction, which is potentially valuable for the future purpose, is the reaction of $N, N^{\prime}-$ dimethylformamide dimethyl acetal (DMFDMA) with 1, 3-dicarbonyl compounds 1 to give enamines $2[2,4]$.
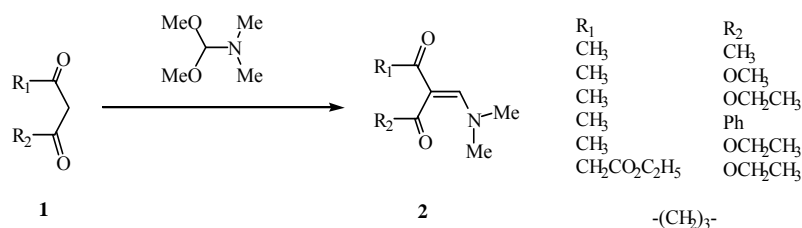

We have reported that enamines 2 were used as precursors in the synthesis of pentasubstituted pyridines 3-6 [5-8].
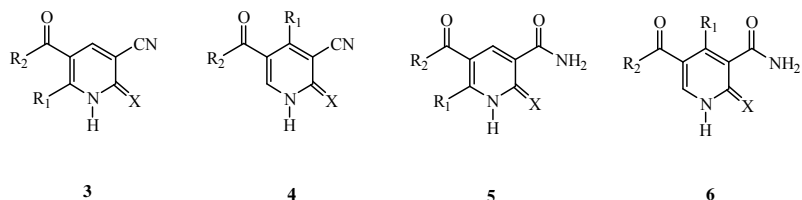

$\mathrm{X}=\mathrm{O}, \mathrm{S}, \mathrm{C}(\mathrm{CN})_{2}$

Moreover, we have reported that $N, N$ '-dimethylformamide dimethyl acetal (DMFDMA) is potentially valuable as a building block for heterocyclic synthesis [9] and used for the synthesis of 1,4-pyrazine-2,5-diones 7 [10].

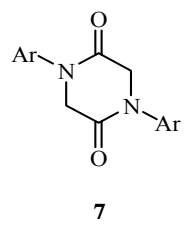

\section{Results and Discussion}

In conjunction with this work we report here the reaction of malononitrile dimer 8 [11] with one mole of $N, N^{\prime}-$ 
dimethylformamide dimethyl acetal (DMFDMA) in dry dioxane afforded only one product that could be formulated as $\mathbf{9}$ or $\mathbf{1 0}$ as result of condensation on either the amino or active methylene group. The structure of the isolated product was elucidated based on the spectral analysis. The ${ }^{1} \mathrm{H}-\mathrm{NMR}$ spectrum shows two singlet signals at $\delta_{\mathrm{H}}=3.2$ and $3.25 \mathrm{ppm}$ corresponding to the two methyl groups of $\mathrm{NMe}_{2}$ moiety, singlet signal at $\delta_{\mathrm{H}}=$ $7.59 \mathrm{ppm}$ corresponding to methylene group or amino group and singlet signal at $\delta_{\mathrm{H}}=7.99 \mathrm{ppm}$ corresponding to methine proton. While we could not differentiate between 9 and 10 by ${ }^{1} \mathrm{H}-\mathrm{NMR}$, DEPT-135 of ${ }^{13} \mathrm{C}-\mathrm{NMR}$ shows a methylene group at $-66.78 \mathrm{ppm}$ which indicates that the isolated product is $\mathbf{9}$ and not $\mathbf{1 0}$. This can be attributed to the fact that the nucleophilicity of the amino group is greater than that of methylene group.

The treatment of malononitrile dimer 8 with two moles of $N, N^{\prime}$-dimethylformamide dimethyl acetal (DMFDMA) afforded amidine $\mathbf{1 1}$ in which $N, N^{\prime}$-dimethylformamide dimethyl acetal (DMFDMA) reacted with both the amino group and the active methylene. The mass spectrum of this compound shows molecular weight at $\mathrm{m} / \mathrm{z} 242$ which corresponds to structure 11. Amidine 11 can also be obtained by treatment of amidine 9 with another one mole of DMFDMA.

The reaction of amidine $\mathbf{1 1}$ with one mole of aromatic amines (1:1) afforded the corresponding pyrimidine derivative 13. while the treatment of amidine $\mathbf{1 1}$ with two moles of aromatic amines (1:2) afforded formamidine $\mathbf{1 4}$ (Scheme 1). This suggests that the isolated pyrimidine 13 was formed through the intermediate 12. The structure of these compounds was confirmed by elemental analysis as well as spectral analysis. The IR spectrum of compound 14 shows the appearance of two bands of $v_{\max }$ at $3286.3 \mathrm{~cm}^{-1}, 3208.2 \mathrm{~cm}^{-1}$ corresponding to two (NH) groups, while the IR spectrum of compound $\mathbf{1 3}$ shows the disappearance of $\mathrm{NH}$ groups. The mass spectrum of compound 14 shows the molecular ion peak at $\mathrm{m} / \mathrm{z} 366$ which is in agreement with the proposed structure $\mathbf{1 4}$.

We expected that the treatment of amidine $\mathbf{1 1}$ with sodium alkoxide (sodium ethoxide, sodium methoxide, sodium $n$-propoxide or sodium isopropoxide) in the corresponding alcohol would afford pyrido[4,3-d]pyrimidine derivatives 16 [12] through the cyclization of the intermediate 15 in which, two molecules of alcohol were added on the two cyano groups. However, the mass spectra of the isolated products shows a molecular weight which does not agree with the expected structure 16. Also the ${ }^{1} \mathrm{H}-\mathrm{NMR}$ spectra shows three exchangeable protons corresponding to $\mathrm{NH}$ and $\mathrm{NH}_{2}$ groups as well as only one aromatic proton. This means that the isolated product is not $\mathbf{1 6}$ and the reaction takes place by another pathway in which the intermediate $\mathbf{1 5}$ is attacked by the alkoxide to give intermediate 17 in which $N, N^{\prime}$-dimethylformamidine moiety is replaced by alkoxide group followed by hydrolysis and cyclization to give 4-alkoxy-5-cyanopyridine-2 $(1 H)$-one-3-carboxylic acid amide 19 . The structure of the isolated product was confirmed by elemental analysis as well as spectral data in which the IR spectra show the presence of $\mathrm{NH}, \mathrm{NH}_{2}$ and cyano group. Also ${ }^{1} \mathrm{H}-\mathrm{NMR}$ spectra show two exchangeable protons for $\mathrm{NH}$ $\& \mathrm{NH}_{2}$ and one aromatic proton. Sodium isopropoxide cannot react with amidine 11. This is due to the fact that the isopropoxide group is a bulkynucleophile. Since it does not replace the $N, N^{\prime}$-dimethylamidine moiety because of the steric hindrance, we could not isolate pyridine isopropoxide derivative (19d) (Scheme 2).

The reaction of malononitrile dimer 8 with diazonium salts of aromatic amines 20a-e furnished the corresponding pyridazine derivatives 21a-e. The structure of the isolated products was confirmed by elemental analysis as well as spectral data. The IR spectra of these compounds show the appearance of amino and imino groups. Also the ${ }^{1} \mathrm{H}-\mathrm{NMR}$ spectra of these compounds 21a-e show the appearance of aromatic protons and two exchangeable broad singlet signals corresponding to $\mathrm{NH}_{2}$ and NH groups.

The pyridazine derivatives 21a-e were found to be a good intermediate for the formation of fused heterocyclic compounds. Reaction of pyridazine derivatives 21a-e with $N, N$ 'dimethylformamide dimethyl acetal (DMFDMA) afforded

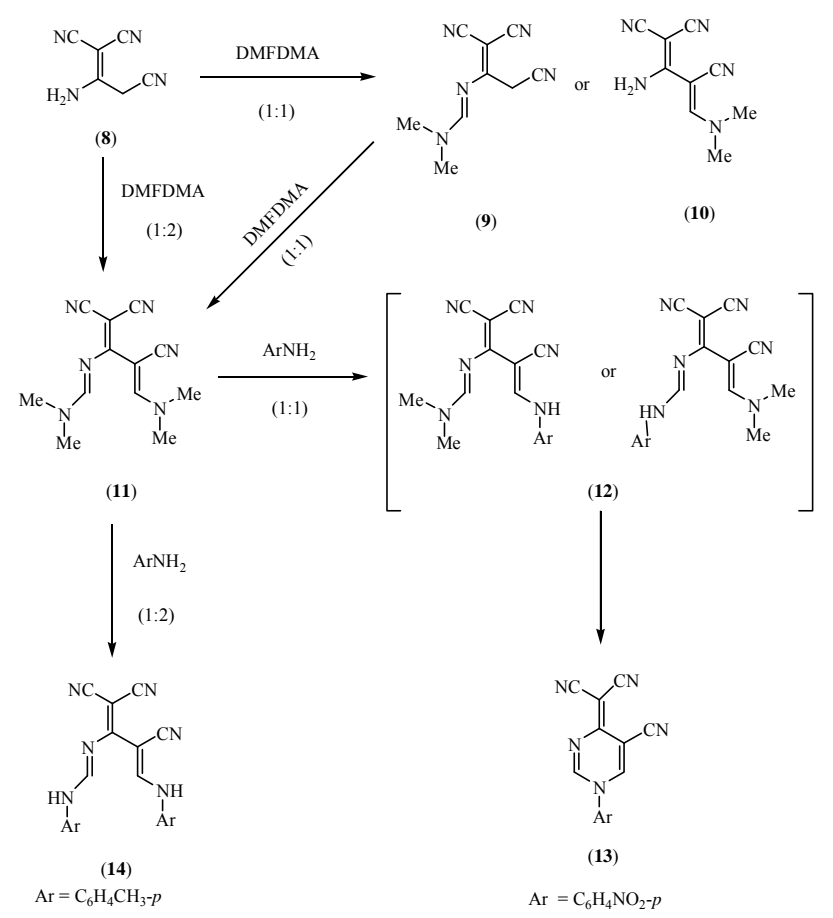

Scheme 1. The reaction and treatment of amidine 11. 


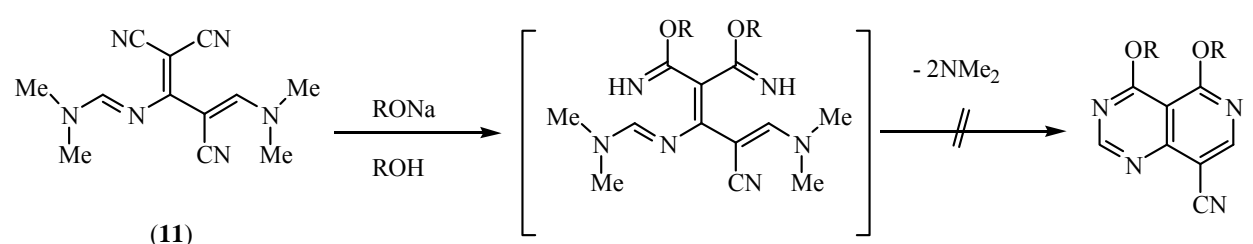

(11)

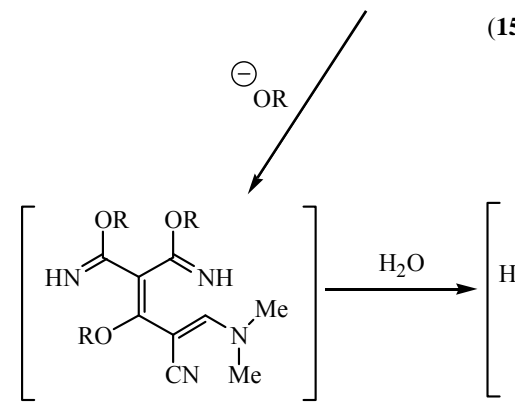

(17)
(15)
(16)

Scheme 2 . The treatment of amidine 11 with sodium alkoxide.

the corresponding amidine 22a-e. IR spectra of these compounds show the disappearance of the amino group, and the ${ }^{1} \mathrm{H}-\mathrm{NMR}$ spectrum of compound $22 \mathbf{b}$ (as an example) shows two singlet signals for 6 protons at $\delta_{\mathrm{H}}=$ $3.10,3.21 \mathrm{ppm}$ corresponding to the two methyl groups of $\mathrm{NMe}_{2}$ moiety, a singlet signal at $\delta_{\mathrm{H}}=8.41 \mathrm{ppm}$ corresponding to $\mathrm{CH}=\mathrm{N}$ proton and the disappearance of the amino group.

Further treatment of pyridazine derivatives $\mathbf{2 1 b}, \mathbf{c}, \mathbf{e}$ with acetic acid in the presence of small amounts of hydrochloric acid afforded the corresponding pyridazinone derivatives 23a-c. The IR spectra of these compounds show disappearance of cyano groups and the appearance of amide carbonyl groups.

Also, the treatment of pyridazine derivatives 21a,b,e with cyanoacetamide afforded pyrido[4,3-c]pyridazine derivatives 24a-c. Consequently, pyridopyridazine derivatives 24a-c were treated with $N, N^{\prime}$-dimethylformamide dimethyl acetal (DMFDMA) to afford the tricyclic heterocycle 25a-c (Scheme 3). The IR spectra of compounds 25a-c show the disappearance of amino group. Also, ${ }^{1} \mathrm{H}-\mathrm{NMR}$ spectra of compounds 25a-c show the disappearance of amino group and the appearance of $(\mathrm{NH})$ group and methine protons at $\delta_{\mathrm{H}}=7.45$ and 8.71 ppm respectively. ${ }^{1}$ H-NMR Spectra of these compounds also show two exchangeable broad signals at $\delta_{\mathrm{H}}=7.45$ and $10.50 \mathrm{ppm}$ The sum of the two integrations of both signals is equivalent to one proton which indicates that these compounds 25a-c may exist as a mixture of three tautomers 25A,B,C.

\section{Experimental}

All melting points are uncorrected. IR spectra were recorded on a Perkin-Elmer 17100 FTIR spectrometer as $\mathrm{KBr}$ disks. NMR spectra were recorded on Bruker AC300 spectrometer at $400 \mathrm{MHz}$ for solutions in $\mathrm{CDCl}_{3}$ or DMSO with tetramethylsilane (TMS) as an internal standard unless otherwise recorded at Department of Chemistry, College of Science, Sultan Qaboos University, P.O. Box 36, Al-Khod23, Oman. Mass spectra were obtained on Finnigan 4500 (low resolution) spectrometers using electron impact (EI) at Micro-analytical Center Cairo University Giza Egypt. N,N'-Dimethylformamide dimethyl acetal (DMFDMA) was purchased from MERCK.

$N$ '-(2,2-Dicyano-1-cyanomethyl-vinyl)- $N, N$-dimethyl -formamidine (9)

In a dry flask, a mixture of malononitrile dimer 8 (1.32 $\mathrm{g}, 10 \mathrm{mmol})$ in dry dioxane $(30 \mathrm{~mL})$ as solvent and $N, N^{\prime}-$ dimethylformamide dimethyl acetal (DMFDMA) (1.32 $\mathrm{ml}, 10 \mathrm{mmol}$ ) was left stirring at room temperature for 24 $\mathrm{h}$ and the solvent was evaporated. The solid product was recovered and recrystallised from ethanol as yellow crystals $(1.63 \mathrm{~g}, 87.17 \%)$; mp. $199^{\circ} \mathrm{C}-201^{\circ} \mathrm{C}$; ${ }^{1} \mathrm{H}-\mathrm{NMR}$ $\left(\mathrm{DMSO}_{6} \mathrm{~d}_{6}\right): \delta 3.20,3.25\left(6 \mathrm{H}, 2 \mathrm{~s}, \mathrm{NMe}_{2}\right), 7.59(2 \mathrm{H}, \mathrm{s}$, $\left.\mathrm{CH}_{2}\right), 7.99(1 \mathrm{H}, \mathrm{s}, \mathrm{CH}) ;{ }^{13} \mathrm{C}-\mathrm{NMR}$ (DMSO-d $\left.{ }_{6}\right): \delta 44.93$, $47.94\left(2 \mathrm{CH}_{3}\right), 69.00\left(\mathrm{CH}_{2}\right), 158.0(\mathrm{CH}), 118.5,169.21$ (C free of hydrogen); DEPT-135 $\delta+38.72,+47.51$ $\left(2 \mathrm{CH}_{3}\right),-66.78\left(\mathrm{CH}_{2}\right),+157.20(\mathrm{CH})$; Anal. Calcd for $\mathrm{C}_{9} \mathrm{H}_{9} \mathrm{~N}_{5}$ (187.21): C, 57.74; H, 4.85; N, 37.41. Found: C, $57.55 ; \mathrm{H}, 4.78 ; \mathrm{N}, 37.22$. 


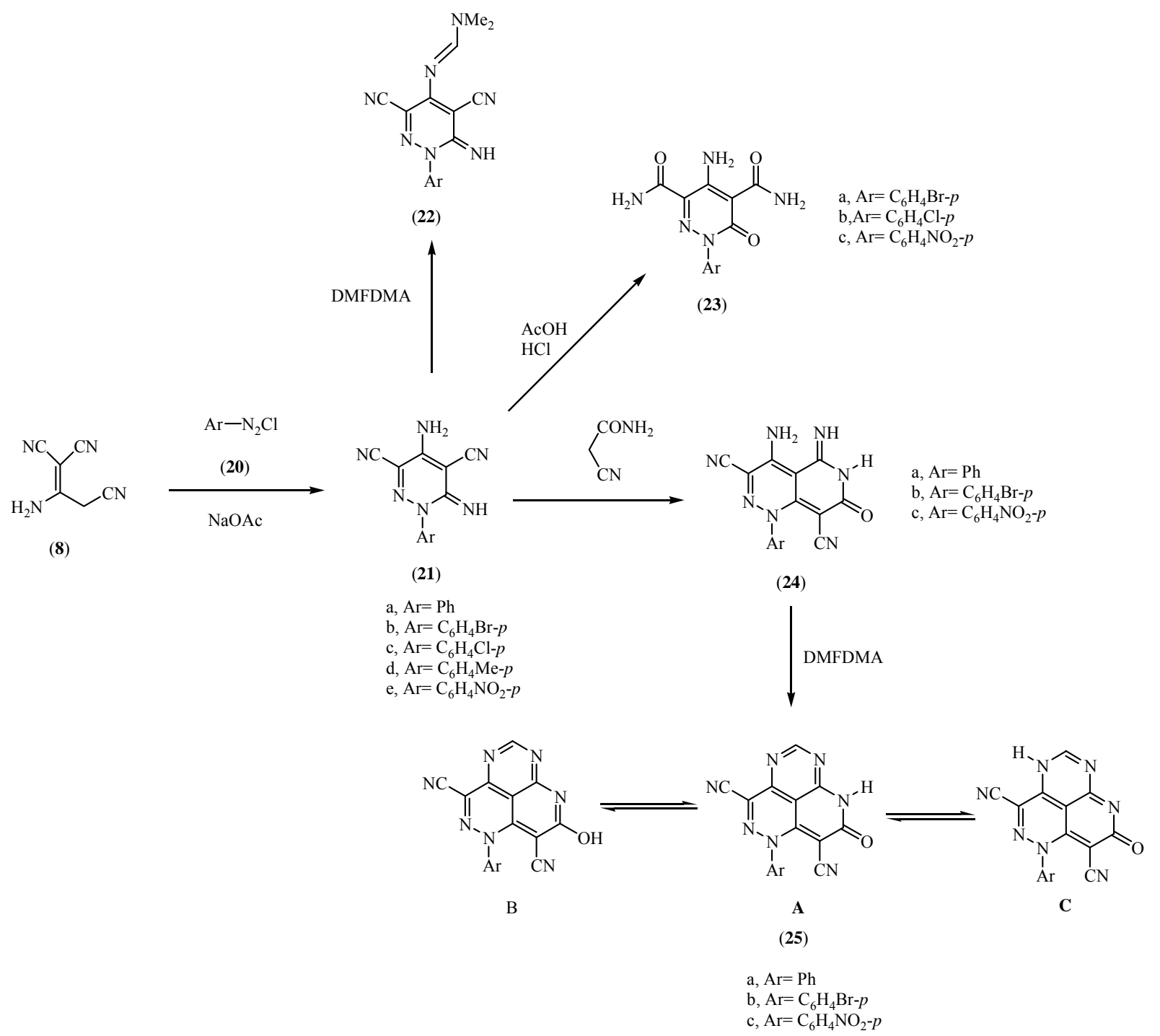

Scheme 3. The treatment of pyridazine derivatives 21a,b,e with cyanoacetamide.

$N^{\prime}$-[2,2-Dicyano-1-(1-cyano-2-dimethylamino-vinyl)vinyl]- $N, N$-dimethyl-formamidine (11)

(A) In a dry flask a mixture of malononitrile dimer 8 $(1.32 \mathrm{~g}, 10 \mathrm{mmol})$ in dry dioxane $(30 \mathrm{~mL})$ as solvent and $N, N^{\prime}$-dimethylformamide dimethyl acetal (DMFDMA) $(2.64 \mathrm{~mL}, 20 \mathrm{mmol})$ was left stirring at room temperature for $24 \mathrm{~h}$ and the solvent was evaporated. The solid product was recovered and recrystallised from ethanol as yellow crystals $(2.1 \mathrm{~g}, 86.4 \%), \mathrm{Mp} .149^{\circ} \mathrm{C}-151^{\circ} \mathrm{C}$; (B) In dry flask a mixture of $N^{\prime}$-(2,2-dicyano-1-cyanomethylvinyl)- $N, N$-dimethylformamidine $9(1.87 \mathrm{~g}, 10 \mathrm{mmol})$ in dry dioxane $(30 \mathrm{~mL})$ as solvent and $N, N^{\prime}$-dimethylformamide dimethyl acetal (DMFDMA) (1.32 mL, $10 \mathrm{mmol})$ was left stirring at room temperature for $24 \mathrm{~h}$ and the solvent was evaporated. The solid product was recovered and recrystallised from ethanol as yellow crystals $(1.9 \mathrm{~g}$,
79.34\%); mp. and mmp. $149^{\circ} \mathrm{C}-151^{\circ} \mathrm{C} ;{ }^{1} \mathrm{H}-\mathrm{NMR}$ $\left(\right.$ DMSO- $\left._{6}\right): \delta 3.06,3.19,3.26,3.34\left(12 \mathrm{H}, 4 \mathrm{~s}, 2 \mathrm{NMe}_{2}\right)$, 7.67, 8.07 (2H, 2s, 2CH); MS (EI) ${ }^{+}: \mathrm{m} / \mathrm{z} 242(90.7 \%) \mathrm{M}^{+}$; Anal. Calcd for $\mathrm{C}_{12} \mathrm{H}_{14} \mathrm{~N}_{6}(242.29)$ : C, 59.49; H, 5.82; N, 34.69. Found: C, 59.31; H, 5.76; N, 34.48.

\section{2-[5-Cyano-1-(4-nitro-phenyl)-1H-pyrimidin-4-ylide} ne]-malononitrile (13)

In a dry flask a mixture of $N^{\prime}$-[2,2-dicyano-1-(1-cyano-2-dimethylamino-vinyl)-vinyl]- $N, N$-dimethyl-formam idine $11(2.42 \mathrm{~g}, 10 \mathrm{mmol})$ in dry xylene $(30 \mathrm{~mL})$ as solvent and 4-nitroanilne (2.9 g, $10 \mathrm{mmol})$ was refluxed for two hours, cooled, and the solvent was evaporated. The solid product was recovered and recrystallised from ethanol as brown crystals $(2.12 \mathrm{~g}, 73.10 \%)$; mp. $206^{\circ} \mathrm{C}-208^{\circ} \mathrm{C}$; IR $(\mathrm{KBr})$ v $2195.2 \mathrm{~cm}^{-1}(\mathrm{CN})$; Anal. Calcd. for $\mathrm{C}_{14} \mathrm{H}_{6} \mathrm{~N}_{6} \mathrm{O}_{2}$ (290.24): C, 57.94; H, 2.08; N, 28.96. Found: C, 57.76; 


\section{$\mathrm{H}, 2.02 ; \mathrm{N}, 28.79$.}

$N$-[2,2-Dicyano-1-(1-cyano-2-p-tolylamino-vinyl)-vin $y l]-N$ '-p-tolyl-formamidine (14)

In a dry flask a mixture of $N^{\prime}$-[2,2-dicyano-1-(1-cyano-2-dimethylamino-vinyl)-vinyl]- $N, N$-dimethyl-formam idine $11(2.42 \mathrm{~g}, 10 \mathrm{mmol})$ in dry xylene $(30 \mathrm{~mL})$ as solvent and $p$-toluidine $(2.14 \mathrm{~g}, 20 \mathrm{mmol})$ was refluxed for two hours, cooled and the solvent was evaporated. The solid product was recovered and recrystallised from ethanol as dark brown crystals $(2.61 \mathrm{~g}, 71.31 \%)$; mp. $289^{\circ} \mathrm{C}$ $291^{\circ} \mathrm{C}$; ${ }^{1} \mathrm{H}-\mathrm{NMR}\left(\mathrm{DMSO}_{6}\right)$ ) $\delta \quad 2.12,2.24(6 \mathrm{H}, 2 \mathrm{~s}$, $\left.2 \mathrm{CH}_{3}\right), 6.42,6.82(8 \mathrm{H}, 2 \mathrm{~d}, \mathrm{Ar}-\mathrm{AB}), 7.05,7.09(2 \mathrm{H}, 2 \mathrm{~s}$, $2 \mathrm{NH}), 7.54,7.56(2 \mathrm{H}, 2 \mathrm{~s}, 2 \mathrm{CH})$; IR ( $\mathrm{KBr})$ v 3286.3, $3208.2(2 \mathrm{NH}), 2225.2,2204.3 \mathrm{~cm}^{-1}(3 \mathrm{CN})$; MS (EI) ${ }^{+}$: $\mathrm{m} / \mathrm{z} 366(10.7 \%) \mathrm{M}^{+}$; Anal. Calcd. for $\mathrm{C}_{22} \mathrm{H}_{18} \mathrm{~N}_{6}$ (366.43): C, 72.11; H, 4.95; N, 22.93. Found: C, 71.92; H, 4.84; N, 22.70 .

\section{General procedure for the preparation of compounds 19a-c}

A mixture of Compound 11 (10 mmol) and sodium alkoxide $(10 \mathrm{mmol})$ in corresponding alcohol $(30 \mathrm{~mL})$ was refluxed for two hours. The mixture was left to cool then poured onto ice cold water. The solid product was recovered by filtration and recrystallised from ethanol.

5-Cyano-4-ethoxy-2-oxo-1,2-dihydro-pyridine-3-carb oxylic acid amide (19a):

Obtained from $11(2.42 \mathrm{~g}, 10 \mathrm{mmol})$ with sodium ethoxide (Na $0.23 \mathrm{~g}$, EtOH $30 \mathrm{~mL}, 10 \mathrm{mmol}) ; \mathrm{mp} .219^{\circ} \mathrm{C}$ $221^{\circ} \mathrm{C}$ as brown crystals (1.46 g, 70.53\%); ${ }^{1} \mathrm{H}-\mathrm{NMR}$ (DMSO- $\left.d_{6}\right): \delta 1.28,1.32,1.35\left(3 \mathrm{H}, \mathrm{t}, \mathrm{CH}_{3}\right), 4.36,4.39$, 4.43, $4.47\left(2 \mathrm{H}, \mathrm{q}, \mathrm{CH}_{2}\right), 7.49(1 \mathrm{H}, \mathrm{s}, \mathrm{NH}), 8.05(2 \mathrm{H}, \mathrm{s}$, $\mathrm{NH}_{2}$, br), 8.47 (1H, s, ring-H); MS (EI) ${ }^{+}: \mathrm{m} / \mathrm{z} 207(39.9 \%)$ $\mathrm{M}^{+}$; Anal. Calcd. for $\mathrm{C}_{9} \mathrm{H}_{9} \mathrm{~N}_{3} \mathrm{O}_{3}$ (207.19): $\mathrm{C}, 52.17 ; \mathrm{H}$, 4.38; N, 20.28. Found: C, 52.02; H, 4.25; N, 20.03.

5-Cyano-4-methoxy-2-oxo-1,2-dihydro-pyridine-3-ca rboxylic acid amide (19b):

Obtained from $11(2.42 \mathrm{~g}, 10 \mathrm{mmol})$ with sodium methoxide (Na $0.23 \mathrm{~g}, \mathrm{MeOH} 30 \mathrm{~mL}, 10 \mathrm{mmol}) ; \mathrm{mp} .229^{\circ} \mathrm{C}$ $231^{\circ} \mathrm{C}$ as brown crystals $(1.43 \mathrm{~g}, 74.09 \%)$; IR $(\mathrm{KBr}) \mathrm{v}$ 3383.7, $3350.9\left(\mathrm{NH}_{2}\right), 3237.5(\mathrm{NH}), 2230.1(\mathrm{CN})$, $1670.5 \mathrm{~cm}^{-1}(\mathrm{C}=\mathrm{O})$; Anal. Calcd. for $\mathrm{C}_{8} \mathrm{H}_{7} \mathrm{~N}_{3} \mathrm{O}_{3}(193.16)$ : C, 49.75; H, 3.65; N, 21.75. Found: C, 49.48; H, 3.56; N, 21.62 .

5-Cyano-2-oxo-4-propoxy-1,2-dihydro-pyridine-3-car boxylic acid amide (19c):

Obtained from 11 (2.42 g, 10mmol) with sodium n-propoxide ( $\mathrm{Na} 0.23 \mathrm{~g}$, n-propanol $30 \mathrm{~mL}, 10 \mathrm{mmol}$ ); mp. $210^{\circ} \mathrm{C}-212^{\circ} \mathrm{C}$ as yellow crystals $(1.91 \mathrm{~g}, 86.43 \%)$; IR (KBr) v 3325.64, $3202.22\left(\mathrm{NH}_{2}\right)$ and $(\mathrm{NH}), 2214.84$ (CN), $1659 \mathrm{~cm}^{-1}(\mathrm{C}=\mathrm{O})$; MS (EI) : m/z 219 (31.3\%) [M-2] ; Anal. Calcd. for $\mathrm{C}_{10} \mathrm{H}_{11} \mathrm{~N}_{3} \mathrm{O}_{3}$ (221.22): C, 54.30; H, 5.01; N, 18.99. Found: C, 54.14; H, 4.90; N, 18.76.

General procedure for the preparation of compounds 21a-e

A mixture of ice cold diazonium salts of aromatic amines 20 [conc. $\mathrm{HCl}(20 \mathrm{~mL})$ added to aromatic amine (10 $\mathrm{mmol})$, cooled then added sodium nitrite $(0.69 \mathrm{~g}, 10$ $\mathrm{mmol})$ ] was added to malononitrile dimer $8(1.32 \mathrm{~g}, 10$ $\mathrm{mmol})$ in ethanol $(30 \mathrm{~mL})$ as solvent in presence of sodium acetate. The precipitate was collected by filtration and recrystallised from ethanol.

4-Amino-6-imino-1-phenyl-1,6-dihydro-pyridazine-3, 5-dicarbonitrile (21a):

Obtained from malononitrile dimer $8(1.32 \mathrm{~g}, 10 \mathrm{mmol})$ and aniline $(0.93 \mathrm{~g}, 10 \mathrm{mmol}) ; \mathrm{mp} .>300^{\circ} \mathrm{C}$ as yellow crystals $(2.20 \mathrm{~g}, 93.22 \%) ;{ }^{1} \mathrm{H}-\mathrm{NMR}$ (DMSO- $\left.d_{6}\right): \delta 7.63$ - $8.04(5 \mathrm{H}, \mathrm{m}, \mathrm{Ar}), 8.85\left(2 \mathrm{H}, \mathrm{s}, \mathrm{NH}_{2}, \mathrm{br}\right), 9.4(1 \mathrm{H}, \mathrm{s}, \mathrm{NH}$, br); IR (KBr) v 3432.2, $3333.8\left(\mathrm{NH}_{2}\right), 3306(\mathrm{NH}), 2207$ (CN); Anal. Calcd for $\mathrm{C}_{12} \mathrm{H}_{8} \mathrm{~N}_{6}$ (236.24): C, 61.01; H, 3.41 ; N, 35.57. Found: C, 60.83; H, 3.27; N, 35.35.

4-Amino-1-(4-bromo-phenyl)-6-imino-1,6-dihydro-py ridazine-3,5-dicarbonitrile (21b):

Obtained from malo- nonitrile dimer 8 (1.32 g, 10 $\mathrm{mmol})$ and 4-bromoaniline (1.725 g, $10 \mathrm{mmol})$; mp. > $300^{\circ} \mathrm{C}$ as yellow crystals $(2.91 \mathrm{~g}, 92.38 \%) ;{ }^{1} \mathrm{H}-\mathrm{NMR}$ $\left(\mathrm{DMSO}_{6}\right): \delta$ 7.41, 8.09 (4H, 2d, Ar-AB), $9.15(2 \mathrm{H}, \mathrm{s}$, $\mathrm{NH}_{2}$, br), 9.94 (1H, s, NH, br); IR (KBr) v 3423, 3337.21 $\left(\mathrm{NH}_{2}\right), 3295.2(\mathrm{NH}), 2210.02 \mathrm{~cm}^{-1}(\mathrm{CN})$; Anal. Calcd. for $\mathrm{C}_{12} \mathrm{H}_{7} \mathrm{BrN}_{6}$ (315.13): C, 45.74; H, 2.24; N, 26.67 . Found: C, 45.52; H, 2.10; N, 26.48.

4-Amino-1-(4-chloro-phenyl)-6-imino-1,6-dihydro-py ridazine-3,5-dicarbonitrile (21c):

Obtained from malononitrile dimer $8(1.32 \mathrm{~g}, 10 \mathrm{mmol})$ and 4-chloroaniline $(1.275 \mathrm{~g}, 10 \mathrm{mmol})$; $\mathrm{mp} .>300^{\circ} \mathrm{C}$ as yellow crystals $(2.49 \mathrm{~g}, 92.22 \%)$; ${ }^{1} \mathrm{H}-\mathrm{NMR}$ (DMSO- $d_{6}$ ): $\delta$ 7.52, 7.98 (4H, 2d, Ar-AB), $9.18\left(2 \mathrm{H}, \mathrm{s}, \mathrm{NH}_{2}, \mathrm{br}\right), 9.88$ $(1 \mathrm{H}, \mathrm{s}, \mathrm{NH}, \mathrm{br})$; IR $(\mathrm{KBr})$ v 3415.6, $3326.61\left(\mathrm{NH}_{2}\right)$, $3308.3(\mathrm{NH}), 2209.06(\mathrm{CN})$; Anal. Calcd. for $\mathrm{C}_{12} \mathrm{H}_{7} \mathrm{ClN}_{6}$ (270.68): C, 53.25; H, 2.61; N, 31.05. Found: C, 53.07; $\mathrm{H}, 2.55 ; \mathrm{N}, 30.89$.

4-Amino-6-imino-1-p-tolyl-1,6-dihydro-pyridazine-3, 5-dicarbonitrile (21d):

Obtained from malononitrile dimer $8(1.32 \mathrm{~g}, 10 \mathrm{mmol})$ and 4-methylaniline $(1.07 \mathrm{~g}, 10 \mathrm{mmol}) ; \mathrm{mp} .>300^{\circ} \mathrm{C}$ as yellow crystals $(2.27 \mathrm{~g}, 90.8 \%)$; ${ }^{1} \mathrm{H}-\mathrm{NMR}$ (DMSO- $d_{6}$ ): $\delta=2.10\left(3 \mathrm{H}, \mathrm{s}, \mathrm{CH}_{3}\right), 7.57,7.69(4 \mathrm{H}, 2 \mathrm{~d}, \mathrm{Ar}-\mathrm{AB}), 9.16$ $\left(2 \mathrm{H}, \mathrm{s}, \mathrm{NH}_{2}\right.$, br), $9.88(1 \mathrm{H}, \mathrm{s}, \mathrm{NH}, \mathrm{br})$; IR $(\mathrm{KBr}) \mathrm{v}$ 3413.25, $3318.8\left(\mathrm{NH}_{2}\right), 3298.5(\mathrm{NH}), 2209.63 \mathrm{~cm}^{-1}(\mathrm{CN})$; Anal. Calcd. for $\mathrm{C}_{13} \mathrm{H}_{10} \mathrm{~N}_{6}(250.26)$ : C, 62.39; H, 4.03; N, 33.58. Found: C, 62.11; H, 3.88; N, 33.37 .

4-Amino-6-imino-1-(4-nitro-phenyl)-1,6-dihydro-pyri dazine-3,5-dicarbonitrile (21e):

Obtained from malononitrile dimer $8(1.32 \mathrm{~g}, 10 \mathrm{mmol})$ and 4-nitroaniline $(1.38 \mathrm{~g}, 10 \mathrm{mmol})$; mp. $>300^{\circ} \mathrm{C}$ as brown crystals $(2.57 \mathrm{~g}, 91.46 \%)$; ${ }^{1} \mathrm{H}-\mathrm{NMR}\left(\mathrm{DMSO}-d_{6}\right)$ : $\delta=7.58,7.72(4 \mathrm{H}, 2 \mathrm{~d}, \mathrm{Ar}-\mathrm{AB}), 9.23\left(2 \mathrm{H}, \mathrm{s}, \mathrm{NH}_{2}, \mathrm{br}\right)$, 
9.89 (1H, s, NH, br); IR (KBr) v 3433.2, $3340.25\left(\mathrm{NH}_{2}\right)$, $3300.5(\mathrm{NH}), 2211.03 \mathrm{~cm}^{-1}(\mathrm{CN})$; Anal. Calcd. for $\mathrm{C}_{12} \mathrm{H}_{7} \mathrm{~N}_{7} \mathrm{O}_{2}$ (281.24): C, 51.25; H, 2.51; N, 34.86. Found: C, 51.04; H, 2.43; N, 34.69.

General procedure for the preparation of compounds 22a-e

Compound 21 (10 mmol) and $N, N^{\prime}$-dimethylformamide dimethyl acetal (DMFDMA) (10 mmol) in dry dioxane $(30 \mathrm{~mL})$ was refluxed for two hours, cooled and evaporated. The precipitate was collected by filtration and recrystallised from ethanol.

N'-(3,5-Dicyano-6-imino-1-phenyl-1,6-dihydro-pyrid azin-4-yl)- $N, N$-dimethyl-formamidine (22a):

Obtained from Compound 21a (2.36 g, $10 \mathrm{mmol})$ with $N, N$ '-dimethylformamide dimethyl acetal (DMFDMA) (1.32 mL, $10 \mathrm{mmol}$ ); mp. $203^{\circ} \mathrm{C}-205^{\circ} \mathrm{C}$ as yellow crystals $(2.24 \mathrm{~g}, 76.98 \%) ;{ }^{1} \mathrm{H}-\mathrm{NMR}$ (DMSO- $\left.d_{6}\right): \delta 3.29$, $3.42\left(6 \mathrm{H}, 2 \mathrm{~s}, \mathrm{NMe}_{2}\right), 6.85$ (1H, s, NH, br), $7.34-7.94$ (5H, m, Ar), 8.27 (1H, s, CH); IR (KBr) v $3306.9(\mathrm{NH})$, $2208.9 \mathrm{~cm}^{-1}(\mathrm{CN})$; Anal. Calcd for $\mathrm{C}_{15} \mathrm{H}_{13} \mathrm{~N}_{7}$ (291.32): $\mathrm{C}$, 61.85; H, 4.50; N, 33.66. Found: C, 61.63; H, 4.37; N, 33.49 .

N'-[1-(4-Bromo-phenyl)-3,5-dicyano-6-imino-1,6-dih ydro-pyridazin-4-yl]- $N, N$-dimethyl-formamidine (22b):

Obtained from Compound 21b (3.15 g, $10 \mathrm{mmol})$ with $N, N$ '-dimethylformamide dimethyl acetal (DMFDMA) (1.32 mL, $10 \mathrm{mmol})$; mp. $209^{\circ} \mathrm{C}-211^{\circ} \mathrm{C}$ as deep brown crystals $(2.85 \mathrm{~g}, 77.03 \%)$; ${ }^{1} \mathrm{H}-\mathrm{NMR}\left(\mathrm{DMSO}-d_{6}\right): \delta 3.10$, $3.21\left(6 \mathrm{H}, 2 \mathrm{~s}, \mathrm{NMe}_{2}\right), 6.98(1 \mathrm{H}, \mathrm{s}, \mathrm{NH}, \mathrm{br}), 7.46,7.72(4 \mathrm{H}$, 2d, Ar-AB), 8.41 (1H, s, CH); IR (KBr) v $3302.8(\mathrm{NH})$, $2202 \mathrm{~cm}^{-1}(\mathrm{CN})$; Anal. Calcd. for $\mathrm{C}_{15} \mathrm{H}_{12} \mathrm{BrN}_{7}$ (370.21): C, 48.67; H, 3.27; N, 26.48. Found: C, 48.44; H, 3.12; N, 26.22 .

N'-[1-(4-Chloro-phenyl)-3,5-dicyano-6-imino-1,6-dih ydro-pyridazin-4-yl]-N,N-dimethyl-formamidine (22c):

Obtained from Compound 21c $(2.7 \mathrm{~g}, 10 \mathrm{mmol})$ with $N, N$ '-dimethylformamide dimethyl acetal (DMFDMA) $(1.32 \mathrm{~mL}, 10 \mathrm{mmol}) \mathrm{mp} .197^{\circ} \mathrm{C}-199^{\circ} \mathrm{C}$ as brown crystals $(2.40 \mathrm{~g}, 73.85 \%)$; ${ }^{1} \mathrm{H}-\mathrm{NMR}$ (DMSO- $\left.d_{6}\right): \delta 3.22$, $3.31\left(6 \mathrm{H}, 2 \mathrm{~s}, \mathrm{NMe}_{2}\right), 6.86(1 \mathrm{H}, \mathrm{s}, \mathrm{NH}, \mathrm{br}), 7.34,7.68(4 \mathrm{H}$, 2d, Ar-AB), 8.46 (1H, s, CH); IR (KBr) v $3312.8(\mathrm{NH})$, $2213.8 \mathrm{~cm}^{-1}(\mathrm{CN})$; Anal. Calcd. for $\mathrm{C}_{15} \mathrm{H}_{12} \mathrm{ClN}_{7}$ (325.76): C, 55.31; H, 3.71; N, 30.10. Found: C, 55.15; H, 3.60; N, 29.93.

N'-(3,5-Dicyano-6-imino-1-p-tolyl-1,6-dihydro-pyrid azin-4-yl)- $N, N$-dimethyl-formamidine (22d):

Obtained from Compound 21d (2.5 g, $10 \mathrm{mmol})$ with $N, N$ '-dimethylformamide dimethyl acetal (DMFDMA) (1.32 mL, $10 \mathrm{mmol})$; mp. $221^{\circ} \mathrm{C}-223^{\circ} \mathrm{C}$ as yellow crystals $(2.34 \mathrm{~g}, 76.72 \%) ;{ }^{1} \mathrm{H}-\mathrm{NMR}$ (DMSO- $\left.d_{6}\right): \delta 2.35(3 \mathrm{H}$, s, $\left.\mathrm{CH}_{3}\right), 3.08,3.20\left(6 \mathrm{H}, 2 \mathrm{~s}, \mathrm{NMe}_{2}\right), 6.65(1 \mathrm{H}, \mathrm{s}, \mathrm{NH}, \mathrm{br})$, 7.40, 7.82 (4H, 2d, Ar-AB), 8.45 (1H, s, CH); IR (KBr) v $3301.8(\mathrm{NH}), 2209.7 \mathrm{~cm}^{-1}(\mathrm{CN})$; Anal. Calcd. for
$\mathrm{C}_{16} \mathrm{H}_{15} \mathrm{~N}_{7}$ (305.34): C, 62.94; H, 4.95; N, 32.11. Found:

C, 62.75; H, 4.84; N, 31.90.

$N^{\prime}$-[3,5-Dicyano-6-imino-1-(4-nitro-phenyl)-1,6-dihy dro-pyridazin-4-yl]- $N, N$-dimethyl-formamidine (22e):

Obtained from Compound $21 \mathrm{e}(2.81 \mathrm{~g}, 10 \mathrm{mmol})$ with $N, N$ '-dimethylformamide dimethyl acetal (DMFDMA) $(1.32 \mathrm{~mL}, 10 \mathrm{mmol})$; mp. $223^{\circ} \mathrm{C}-225^{\circ} \mathrm{C}$ as deep brown crystals $(2.82 \mathrm{~g}, 83.93 \%) ;{ }^{1} \mathrm{H}-\mathrm{NMR}\left(\mathrm{DMSO}-d_{6}\right): \delta 3.12$, $3.28\left(6 \mathrm{H}, 2 \mathrm{~s}, \mathrm{NMe}_{2}\right), 7.40(1 \mathrm{H}, \mathrm{s}, \mathrm{NH}, \mathrm{br}), 7.88,8.39(4 \mathrm{H}$, 2d, Ar-AB), 8.50 (1H, s, CH); IR (KBr) v 3290.8 (NH), $2207.6 \mathrm{~cm}^{-1}(\mathrm{CN})$; Anal. Calcd. for $\mathrm{C}_{15} \mathrm{H}_{12} \mathrm{~N}_{8} \mathrm{O}_{2}$ (336.32): C, 53.57; H, 3.60; N, 33.32. Found: C, 53.35; H, 3.48; N, 33.15 .

General procedure for the preparation of compounds 23a-c

Compound $21(10 \mathrm{~mol})$ in acetic acid $(20 \mathrm{~mL})$ and hydrochloric acid $(3 \mathrm{~mL})$ was refluxed for four hours, cooled, and poured onto ice cold water.. The precipitate which formed was recovered by filtration and recrystallised from ethanol.

4-Amino-1-(4-bromo-phenyl)-6-oxo-1,6-dihydro-pyri dazine-3,5-dicarboxylic acid diamide (23a):

Obtained from Compound 21b (3.15 g, $10 \mathrm{mmol})$; mp. $>300^{\circ} \mathrm{C}$ as deep brown crystals (2.65 g, 75.28\%); ${ }^{1} \mathrm{H}-\mathrm{NMR}\left(\mathrm{DMSO}-d_{6}\right): \delta 7.82 ; 8.64(4 \mathrm{H}, 2 \mathrm{~d}, \mathrm{Ar}-\mathrm{AB})$, $7.98\left(2 \mathrm{H}, \mathrm{s}, \mathrm{NH}_{2}\right), 9.79\left(2 \mathrm{H}, \mathrm{s}, \mathrm{NH}_{2}, \mathrm{br}\right)$; IR $(\mathrm{KBr}) \mathrm{v}$ 3376.3, $3314.6\left(\mathrm{NH}_{2}\right), 1701,1663.9 \mathrm{~cm}^{-1}(\mathrm{C}=\mathrm{O})$; Anal. Calcd. for $\mathrm{C}_{12} \mathrm{H}_{10} \mathrm{BrN}_{5} \mathrm{O}_{3}$ (352.15): C, 40.93; H, 2.86; N, 19.89. Found: C, 40.71; H, 2.74; N, 19.60.

4-Aamino-1-(4-chloro-phenyl)-6-oxo-1,6-dihydro-pyr idazine-3,5-dicarboxylic acid diamide (23b):

Obtained from Compound 21c (2.7 g, $10 \mathrm{mmol})$; mp. $>300^{\circ} \mathrm{C}$ as brown crystals $(2.23 \mathrm{~g}, 72.64 \%) ;{ }^{1} \mathrm{H}-\mathrm{NMR}$ (DMSO- $\left.d_{6}\right): \delta 7.78,8.40(4 \mathrm{H}, 2 \mathrm{~d}, \mathrm{Ar}-\mathrm{AB}), 8.01(2 \mathrm{H}, \mathrm{s}$, $\left.\mathrm{NH}_{2}\right), 9.85\left(2 \mathrm{H}, \mathrm{s}, \mathrm{NH}_{2}\right.$, br); IR (KBr) v 3314.5, 3197.7 $\left(\mathrm{NH}_{2}\right), 1697.7,1630 \mathrm{~cm}^{-1}(\mathrm{C}=\mathrm{O})$; Anal. Calcd. for $\mathrm{C}_{12} \mathrm{H}_{10} \mathrm{ClN}_{5} \mathrm{O}_{3}$ (307.70): C, 46.84; H, 3.28; N, 22.76. Found: C, 46.59; H, 3.13; N, 22.61.

4-Amino-1-(4-nitro-phenyl)-6-oxo-1,6-dihydro-pyrid azine-3,5-dicarboxylic acid diamide (23c):

Obtained from Compound 21e (2.81 g, $10 \mathrm{mmol})$; $\mathrm{mp}$. $>300^{\circ} \mathrm{C}$ as brownish crystals $(2.42 \mathrm{~g}, 76.10 \%) ;{ }^{1} \mathrm{H}-$ NMR (DMSO- $\left.d_{6}\right): \delta 7.90,8.55(4 \mathrm{H}, 2 \mathrm{~d}, \mathrm{Ar}-\mathrm{AB}), 8.30$ $\left(2 \mathrm{H}, \mathrm{s}, \mathrm{NH}_{2}\right), 9.60\left(2 \mathrm{H}, \mathrm{s}, \mathrm{NH}_{2}\right.$, br); IR (KBr) v 3381.0, $3272.0\left(\mathrm{NH}_{2}\right), 1691.9,1654.7 \mathrm{~cm}^{-1}(\mathrm{C}=\mathrm{O})$; Anal. Calcd. for $\mathrm{C}_{12} \mathrm{H}_{10} \mathrm{~N}_{6} \mathrm{O}_{5}$ (318.25): C, 45.29; H, 3.17; N, 26.41. Found: C, 45.03; H, 3.06; N, 26.24.

General procedure for the preparation of compounds 24a-c

A mixture of Compound 21 (10 mmol) and cyanoacetamide $(10 \mathrm{mmol})$ in ethanol $(30 \mathrm{~mL})$ and $3-5$ drops of piperidine as a base was refluxed for two hours, cooled, and poured onto ice cold water. The precipitate was re- 
covered by filtration and recrystallised from ethanol.

4-Amino-5-imino-7-oxo-1-phenyl-1,5,6,7-tetrahydropyrido[4,3-c]pyridazine-3,8-dicarbonitrile (24a):

Obtained from Compound 21a $(2.36 \mathrm{~g}, 10 \mathrm{mmol})$ with cyanoacetamide $(0.84 \mathrm{~g}, 10 \mathrm{mmol}) ; \mathrm{mp} .>300^{\circ} \mathrm{C}$ as brown crystals $(2.22 \mathrm{~g}, 73.27 \%)$; ${ }^{1} \mathrm{H}-\mathrm{NMR}$ (DMSO- $d_{6}$ ): $\delta 7.23\left(2 \mathrm{H}, \mathrm{s}, \mathrm{NH}_{2}\right), 7.61,10.52(2 \mathrm{H}, 2 \mathrm{~s}, 2 \mathrm{NH}, \mathrm{br}), 7.64-$ $8.12(5 \mathrm{H}, \mathrm{m}, \mathrm{Ar}) ; \mathrm{IR}(\mathrm{KBr})$ v 3420.6, $3382.7\left(\mathrm{NH}_{2}\right), 3343$ $\mathrm{cm}^{-1}(\mathrm{NH}), 2210.5 \mathrm{~cm}^{-1}(\mathrm{CN}), 1683.2 \mathrm{~cm}^{-1}(\mathrm{C}=\mathrm{O})$; Anal. Calcd. for $\mathrm{C}_{15} \mathrm{H}_{9} \mathrm{~N}_{7} \mathrm{O}$ (303.29): C, 59.41; H, 2.99; N, 32.33. Found: C, 59.22; H, 2.87; N, 32.19 .

4-Amino-1-(4-bromo-phenyl)-5-imino-7-oxo-1,5,6,7-t etrahydro-pyrido[4,3-c]pyridazine-3,8-dicarbonitrile (24b):

Obtained from Compound $21 \mathrm{~b}(3.15 \mathrm{~g}, 10 \mathrm{mmol})$ with cyanoacetamide $(0.84 \mathrm{~g}, 10 \mathrm{mmol}) ; \mathrm{mp} .179^{\circ} \mathrm{C}-181^{\circ} \mathrm{C}$ as brown crystals $(2.83 \mathrm{~g}, 74.08 \%)$; ${ }^{1} \mathrm{H}-\mathrm{NMR}$ (DMSO$\left.d_{6}\right): \delta 6.70\left(2 \mathrm{H}, \mathrm{s}, \mathrm{NH}_{2}\right), 7.68,10.20(2 \mathrm{H}, 2 \mathrm{~s}, 2 \mathrm{NH}, \mathrm{br})$, 7.50, 8.20 (4H, 2d, Ar-AB); IR (KBr) v 3402.0, 3325.1 $\left(\mathrm{NH}_{2}\right), 3175.1(\mathrm{NH}), 2204.8(\mathrm{CN}), 1617.5 \mathrm{~cm}^{-1}(\mathrm{C}=\mathrm{O})$; Anal. Calcd. for $\mathrm{C}_{15} \mathrm{H}_{8} \mathrm{BrN}_{7} \mathrm{O}$ (382.18): C, 47.14; $\mathrm{H}, 2.11$; N, 25.65. Found: C, 46.91; H, 2.02; N, 25.41.

4-Amino-5-imino-1-(4-nitro-phenyl)-7-oxo-1,5,6,7-tet rahydro-pyrido[4,3-c]pyridazine-3,8-dicarbonitrile (24c):

Obtained from Compound 21e $(2.81 \mathrm{~g}, 10 \mathrm{mmol})$ with cyanoacetamide $(0.84 \mathrm{~g}, 10 \mathrm{mmol}) \mathrm{mp} .239^{\circ} \mathrm{C}-241^{\circ} \mathrm{C}$ as brown crystals $(2.39 \mathrm{~g}, 68.68 \%)$; ${ }^{1} \mathrm{H}-\mathrm{NMR}$ (DMSO- $d_{6}$ ): $\delta 7.19\left(2 \mathrm{H}, \mathrm{s}, \mathrm{NH}_{2}\right), 7.55,10.60(2 \mathrm{H}, 2 \mathrm{~s}, 2 \mathrm{NH}, \mathrm{br}), 7.78$, 8.34 (4H, 2d, Ar-AB); IR (KBr) v 3462.0, $3352.3\left(\mathrm{NH}_{2}\right)$, $3228.2(\mathrm{NH}), 2192(\mathrm{CN}), 1630.0 \mathrm{~cm}^{-1}(\mathrm{C}=\mathrm{O})$; Anal. Calcd. for $\mathrm{C}_{15} \mathrm{H}_{8} \mathrm{~N}_{8} \mathrm{O}_{3}$ (348.28): C, 51.73; H, 2.32; N, 32.17. Found: C, 51.50; H, 2.19; N, 32.13.

General procedure for the preparation of compounds 25a-c:

Compound $24(10 \mathrm{mmol})$ and $N, N^{\prime}$-dimethylformamide dimethyl acetal (DMFDMA) $(10 \mathrm{mmol})$ in dry dioxane $(30 \mathrm{~mL})$ was refluxed for two hours, cooled, and evaporated. The precipitate was collected by filtration and recrystallised from ethanol.

8-Oxo-1-phenyl-7,8-dihydro-1H-1,2,4,6,7-pentaaza-p henalene-3,9-dicarbonitrile (25a):

Obtained from Compound 24a (3.03 g, $10 \mathrm{mmol})$ with $N, N$ '-dimethylformamide dimethyl acetal (DMFDMA) $(1.32 \mathrm{~mL}, 10 \mathrm{mmol}) ; \mathrm{mp} .>300^{\circ} \mathrm{C}$ as brown crystals $(2.29$ g, 73.16\%); ${ }^{1} \mathrm{H}-\mathrm{NMR}\left(\mathrm{DMSO}-d_{6}\right): \delta 7.37(1 \mathrm{H}, \mathrm{s}, \mathrm{NH}, \mathrm{br})$, 7.81-8.36 (5H, m, Ar), $8.86(1 \mathrm{H}, \mathrm{s}, \mathrm{CH}), 10.30(1 \mathrm{H}, \mathrm{s}, \mathrm{OH}$, br); IR (KBr) v $3333.7(\mathrm{NH}), 2205.7(\mathrm{CN}), 1629 \mathrm{~cm}^{-1}$ $(\mathrm{C}=\mathrm{O})$; Anal. Calcd. for $\mathrm{C}_{16} \mathrm{H}_{7} \mathrm{~N}_{7} \mathrm{O}(313.28)$ : $\mathrm{C}, 61.34 ; \mathrm{H}$, 2.25; N, 31.30. Found: C, 61.18; H, 2.08; N, 31.16.

1-(4-Bromo-phenyl)-8-oxo-7,8-dihydro-1H-1,2,4,6,7pentaaza-phenalene-3,9-dicarbonitrile (25b):

Obtained from Compound $24 \mathbf{b}$ ( $3.82 \mathrm{~g}, 10 \mathrm{mmol})$ with
$N, N$ '-dimethylformamide dimethyl acetal (DMFDMA) $(1.32 \mathrm{~mL}, 10 \mathrm{mmol}) ; \mathrm{mp} .>300^{\circ} \mathrm{C}$ as deep brown crystals $(2.76 \mathrm{~g}, 70.41 \%) ;{ }^{1} \mathrm{H}-\mathrm{NMR}$ (DMSO- $\left.d_{6}\right): \delta 7.51(1 \mathrm{H}$, s, NH, br), 7.69, 8.28 (4H, 2d, Ar-AB), $8.84(1 \mathrm{H}, \mathrm{s}, \mathrm{CH})$, $10.02(1 \mathrm{H}, \mathrm{s}, \mathrm{OH}, \mathrm{br})$; IR (KBr) v $3326.9(\mathrm{NH}), 2206.1$ $(\mathrm{CN}), 1623 \mathrm{~cm}^{-1}(\mathrm{C}=\mathrm{O})$; Anal. Calcd. for $\mathrm{C}_{16} \mathrm{H}_{6} \mathrm{BrN}_{7} \mathrm{O}$ (392.18) C, 49.00; H, 1.54; N, 25.00. Found: C, 48.79; H, $1.42 ; \mathrm{N}, 24.85$.

1-(4-Nitro-phenyl)-8-oxo-7,8-dihydro-1H-1,2,4,6,7-p entaaza-phenalene-3,9-dicarbonitrile (25c):

Obtained from Compound 24c (3.48 g, $10 \mathrm{mmol})$ with $N, N$ '-dimethylformamide dimethyl acetal (DMFDMA) $(1.32 \mathrm{~mL}, 10 \mathrm{mmol}) ; \mathrm{mp} .>300^{\circ} \mathrm{C}$ as brown crystals $(2.52 \mathrm{~g}, 70.39 \%) ;{ }^{1} \mathrm{H}-\mathrm{NMR}$ (DMSO- $\left.d_{6}\right): \delta 7.45(1 \mathrm{H}, \mathrm{s}$, $\mathrm{NH}, \mathrm{br}), 7.78,8.32(4 \mathrm{H}, 2 \mathrm{~d}, \mathrm{Ar}-\mathrm{AB}), 8.71(1 \mathrm{H}, \mathrm{s}, \mathrm{CH})$, $10.50(1 \mathrm{H}, \mathrm{s}, \mathrm{OH}, \mathrm{br})$; IR $(\mathrm{KBr})$ v $3338.4(\mathrm{NH}), 2202.5$ (CN), $1618 \mathrm{~cm}^{-1}(\mathrm{C}=\mathrm{O})$; Anal. Calcd. for $\mathrm{C}_{16} \mathrm{H}_{6} \mathrm{~N}_{8} \mathrm{O}_{3}$ (358.28): C, 53.64; H, 1.69; N, 31.28. Found: C, 53.41; $\mathrm{H}, 1.58 ; \mathrm{N}, 31.05$.

\section{References}

[1] V. G. Granik, A. M. Zhidkova and R. G. Glushkov, "Advances in the Chemistry of the Acetals of Acid Amides and Lactams," Russian Chemical Reviews, Vol. 46, No. 4, 1977, pp. 361-366. doi:10.1070/RC1977v046n04ABEH002137

[2] R. F. Abdulla and R. S. Brinkmeyer, "The Chemistry of Formamide Acetals," Tetrahedron, Vol. 35, No. 14, 1979, pp. 1675-1735. doi:10.1016/0040-4020(79)88001-1

[3] P. L. Anelli, M. Brocchetta, D. Palano and M. Visigalli, "Mild Conversion of Primary Carboxamides into Carboxylic Esters," Tetrahedron Letters, Vol. 38, No. 13, 1997, pp. 2367-2368. doi:10.1016/S0040-4039(97)00350-X

[4] M. Malesic, A. Krbavcic, A. Golobic, L. Golic and B. Stanovenik, "The Synthesis and Transformation of Ethyl 2-(2-Acetyl-2-benzoyl-1-ethenyl)amino-3-dimethylamino propenoate. A New Synthesis of 2,3,4-Trisubstituted Pyrroles," Journal of Heterocyclic Chemistry, Vol. 34, No. 6, 1997, pp. 1757-1762. doi:10.1002/jhet.5570340619

[5] F. A. Abu-Shanab, A. D. Redhouse, J. R. Thompson and B. J. Wakefield, "Synthesis of 2,3,5,6-Tetrasubstituted Pyridines from Enamines Derived from N,N-Dimethylformamide Dimethyl Acetal," Synthesis, Vol. 5, 1995, pp. 557-560. doi:10.1055/s-1995-3954

[6] F. A. Abu-Shanab, F. M. Aly and B. J. Wakefield, "Synthesis of Substituted Nicotinamides from Enamines Derived from N,N-Dimethylformamide Dimethyl Acetal," Synthesis, Vol. 8, 1995, pp. 923-925. doi:10.1055/s-1995-4039

[7] F. A. Abu-Shanab, A. M. Hessen and S. A. S. Mousa, "Dimethylformamide Dimethyl Acetal in Heterocyclic Synthesis: Synthesis of Polyfunctionally Substituted Pyridine Derivatives as Precursors to Bicycles and Polycy- 
cles," Journal of Heterocyclic Chemistry, Vol. 44, No. 4, 2007, pp. 787-791. doi:10.1002/jhet.5570440406

[8] F. A. Abu-Shanab, M. H. Elnagdi, F. M. Aly and B. J. Wakefield, Journal of the Chemical Society, Perkin Transactions 1, Vol. 1, 1994, pp. 1449-1452.

[9] F. A. Abu-Shanab, S. A. S. Mousa, et al., "Dimethylformamide Dimethyl Acetal as a Building Block in Heterocyclic Synthesis," Journal of Heterocyclic Chemistry, Vol. 46, No. 5, 2009, pp. 801-827. doi:10.1002/jhet.69

[10] F. A. Abu-Shanab, A. El-Harrasi and S. A. S. Mousa, "Synthesis of 1,4-Diaryl-piperazine-2,5-diones: New Behavior of N,N-Dimethylformamide Dimethyl Acetal (DMF
DMA)," Synthetic Communications, Vol. 38, No. 3, 2008, pp. 376-382. doi:10.1080/00397910701767098

[11] R. A. Carboni, D. D. Conffman and E. G. Howard, "Cyanocarbon Chemistry. XI.1 Malononitrile Dimer," Journal of the American Chemical Society, Vol. 80, No. 11, 1958, pp. 2838-2840.

[12] F. A. Abu-Shanab, Y. M. Elkholy and M. H. Elnagdi, "Enaminones as Building Blocks in Organic Synthesis: Synthesis of New Polyfunctional Pyridines, Condensed Pyridines, and Penta Substituted Benzene," Synthetic Communications, Vol. 32, No. 22, 2002, pp. 3493-3502. 